Saher S Gasgoos BDS, MSc (Lect.)

\title{
Caries Severity of Primary Teeth among Kin- dergarten Children in Mosul City Using In- ternational Caries Detection and Assessment System
}

\author{
Dept of Pedod, orthod, and Prev Dentistry \\ College of Dentistry, University of Mosul
}

$$
\begin{aligned}
& \text { الحلاصة } \\
& \text { الأهداف:هدف الدراسة اللى تقيم حدة تسوس الأسنان و علاقته مع عوامل عديدة مثل مستوى تعليم و دخل الأسرة و مراجعة طبيب الأسنان }
\end{aligned}
$$

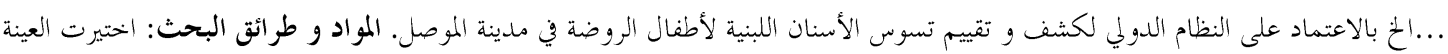

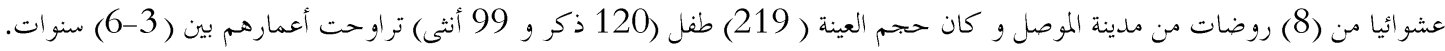

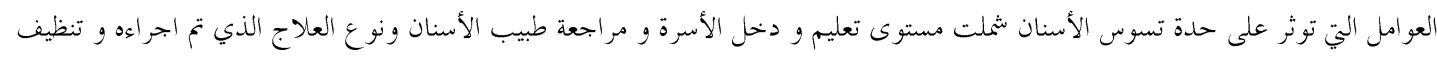

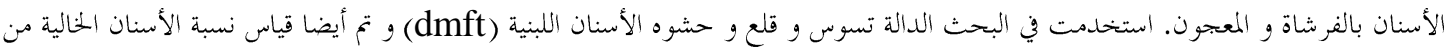

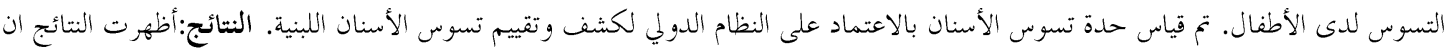

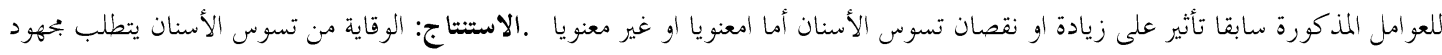

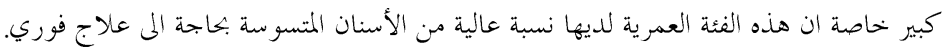

\begin{abstract}
Aims: To assess the severity of dental caries and its relation to risk factors such as parents' education, income level, demographic variables, dental attendance....etc using the International Caries Detection and Assessment System on the primary teeth of kindergarten children in Mosul City Center. Materials and Methods: The study sample was randomly selected from (8) kindergarten in Mosul City. The sample size was (219) child (120 males and 99 females), their ages ranged between (3-6) years. Risk factors considered on caries severity included parent's income level, parent's education, visiting the dentist, types of dental treatment performed and brushing behavior. dmft and the percentage of caries free students were also considered. Caries severity was measured using International Caries Detection and Assessment System. Results: These factors have an effect to increase or decrease caries severity either significantly or not significantly. Conclusion: considerable efforts are required in prevention of dental caries in these children. There is a lot of untreated caries, which needs immediate attention.

Key words: caries severity, dmft, kindergarten, ICDAS.

Gasgoos SS. Caries Severity of Primary Teeth among Kindergarten Children in Mosul City Using International Caries Detection and Assessment System. Al-Rafidain Dent J. 2011; 11(1):183-193.

Received: 17/12/2009 Sent to Referees: $12 / 12 / 2009$

Accepted for Publication: 18/3/2010
\end{abstract}

\section{INTRODUCTION}

Dental caries is one of the most common oral diseases, it is a major problem in dentistry and should receive significant attention from restorative and preventive stand point. $^{(1)}$ It is usually starts and progresses at young age. However, caries experience for primary dentition was decreasing with increasing age, This may be attributed to exfoliation of the primary teeth at older age. ${ }^{(2,3)}$

There is a strong association between low income and education on the one hand and a higher mortality and morbidity of the teeth on the other. ${ }^{(4)}$ Young children from poorer social backgrounds have higher caries levels. ${ }^{(5)}$ Yet in some developing countries, children from higher socioeconomic levels have higher caries experience in primary teeth. This has been attributed to the reason of consuming more refined sugar and cariogenic food than other individuals. ${ }^{(6)}$

Information regarding non cavitated carious lesion in young children provide important additional components of understanding the carious process. Several studies of dental caries in the world have not recorded non cavitated carious lesions in primary teeth. Further, caries preventive programs usually start in schools with 6years-old or older. A survey of the early 
stages of caries development is necessary to set up an appropriate preventive program for children. ${ }^{(7)}$ Hence, this study is focused on non cavitated and cavitated carious lesion, and will assess the prevalence, severity of dental caries and its relation to risk factors such as parents education, income level, demographic variables (such as age, gender), dental attendance....etc using the International Caries Detection and Assessment System on the primary teeth of kindergarten children aged 3-6 years in Mosul City center.

\section{MATERIALS AND METHODS}

The study sample was randomly selected from (8) kindergarten in Mosul City. The sample size was (219) child (120 males and 99 females), their ages ranged between (3-6) years, so that all the deciduous teeth can be examined before their normal exfoliation time. Risk indicators were coded as follow:

1. Demographic variables; age and sex: Group 1: (3-4) years old (males and females); Group 2: (4-5) years old (males and females) and Group 3: (5-6) years old (males and females).

2. Family income monthly in Dinars: Group 1: $(<250000)$; Group 2: $(<500000)$; Group 3(<1000000) and Group 4: (>1000000).

3. Parent's education: Group 1: (Prima- ry school); Group 2: (Secondary School); Group 3: (College) and Group 4: (High education).

4. Last dental visit: Group 1: (Have previous dental attendance) and Group 2: (Did not have previous dental attendance).

5. Reason of dental visit: Group 1: (Pain / treatment); Group 2: (Prevention); Group 3: (Restoration) and Group 4: (Extraction).

6. Teeth brushing: Group 1: (Brush their teeth) and Group 2: (Do not brush their teeth). The (brushing) group was subdivided into (infrequent brushing), (once daily) and (twice daily and more).

A questionnaires related to the previous information were distributed to the children and were completed by their parents. For each factor groups, all children's teeth were examined for the presence and severity of dental caries on the deciduous teeth. Children who had exfoliated anterior teeth were excluded from the study. Dental examination was carried out under natural day light. Instruments used were plane mouth mirror, air syringes, sharp probes to check for surface discontinuity, saliva ejector, cotton and disinfectant solution. Both primary carious lesion (non cavitated) and cavitated lesions were examined. The criteria followed for caries severity of International Caries Detection and Assessment System. ${ }^{(8)}$

\begin{tabular}{|c|l|}
\hline Code & Description \\
\hline 0 & Sound tooth surface \\
\hline 1 & $\begin{array}{l}\text { First visual change in enamel. When seen wet, there is no evidence of any change } \\
\text { in color attributable to carious activity, but after air drying for 5 seconds a carious } \\
\text { opacity is visible }\end{array}$ \\
\hline 2 & $\begin{array}{l}\text { Distinct visual change in enamel, there is a carious opacity or discoloration that is } \\
\text { not consistent with the clinical appearance of sound enamel. This lesion may be } \\
\text { seen directly when viewed from the buccal or lingual direction. When viewed } \\
\text { from occlusal direction, this discoloration may be seen as a shadow confined to } \\
\text { enamel. }\end{array}$ \\
\hline 3 & $\begin{array}{l}\text { Initial brake down in enamel due to caries with no visible dentin. Once dried for 5 } \\
\text { seconds, there is a distinct loss of enamel integrity, viewed from the buccal or } \\
\text { lingual direction. }\end{array}$ \\
\hline 4 & $\begin{array}{l}\text { Non cavitated surface with underlying dark shadow from dentin. This lesion ap- } \\
\text { pears as a shadow of discolored dentin visible through an apparently intact mar- } \\
\text { ginal ridge, buccal or lingual walls of enamel. }\end{array}$ \\
\hline 5 & $\begin{array}{l}\text { Distinct cavity with visible dentin. Cavitation if opaque or discolored enamel with } \\
\text { exposed dentin in the examiner's judgment. }\end{array}$ \\
\hline 6 & $\begin{array}{l}\text { Extensive distinct cavity with visible dentin. Obvious loss of tooth structure, the } \\
\text { extensive cavity may be deep or wide and dentin is clearly visible on both the } \\
\text { walls and at the base. }\end{array}$ \\
\hline
\end{tabular}


Analysis of the data included calculation of percentage, Spearman's correlation coefficient.

\section{RESULTS}

Table (1) shows the distribution of the children according to age and gender.

Table (1): Distribution of the children according to age and gender.

\begin{tabular}{cccccccc}
\hline \multicolumn{2}{c}{ Age group } & \multicolumn{2}{c}{ Males } & \multicolumn{2}{c}{ females } & \multicolumn{2}{c}{ Total } \\
& & No. & $\%$ & No. & $\%$ & No. & $\%$ \\
\hline \multirow{2}{*}{ 3-4 year } & No. & 33 & 55.93 & 26 & 44.07 & 59 & 100 \\
& $\%$ & 27.5 & & 26.26 & & 26.94 & \\
\multirow{4}{*}{ 4-5 year } & No. & 39 & 52.7 & 35 & 47.3 & 74 & 100 \\
& $\%$ & 32.5 & & 35.35 & & 33.79 & \\
5-6 year & No. & 48 & 55.81 & 38 & 44.19 & 86 & 100 \\
& $\%$ & 40 & & 38.39 & & 39.27 & \\
\multirow{4}{*}{ Total } & No. & 120 & 54.79 & 99 & 45.21 & 219 & 100 \\
& $\%$ & 100 & & 100 & & 100 & \\
\hline
\end{tabular}

Table (2) and figure (1) show the relation between gender and dental caries severity for all age groups. It is clear from the table that there were a slight gender differences in the mean dmft scores. This mean was slightly larger for females (4.22) than males (4.12) at age group (4-5y) but the opposite was true regarding the other age groups. Spearman's correlation between gender and caries severity was only significant with (d2) at 0.05 level.

Table (2): ICDAS gender differences.

\begin{tabular}{|c|c|c|c|c|c|c|c|c|c|}
\hline Age / sex & No. & dmft & No.\&\% & d 1 & d 2 & d 3 & d 4 & d 5 & d 6 \\
\hline \multirow{2}{*}{$\begin{array}{l}3-4 \text { y / } \\
\text { males }\end{array}$} & \multirow{2}{*}{33} & \multirow{2}{*}{2.42} & No. & 4 & 18 & 23 & 14 & 13 & 3 \\
\hline & & & $\%$ & 5.33 & 24 & 30.67 & 18.67 & 17.33 & 4 \\
\hline \multirow{2}{*}{$\begin{array}{c}\text { 3-4 y / } \\
\text { females }\end{array}$} & \multirow{2}{*}{26} & \multirow{2}{*}{2.11} & No. & 2 & 12 & 22 & 8 & 7 & 1 \\
\hline & & & $\%$ & 3.85 & 23.08 & 42.31 & 15.38 & 13.46 & 1.92 \\
\hline \multirow{2}{*}{$\begin{array}{l}4-5 \text { y / } \\
\text { males }\end{array}$} & \multirow{2}{*}{39} & \multirow{2}{*}{4.12} & No. & 3 & 17 & 38 & 27 & 54 & 6 \\
\hline & & & $\%$ & 2.07 & 11.72 & 26.21 & 18.62 & 37.24 & 4.14 \\
\hline \multirow{2}{*}{$\begin{array}{c}\text { 4-5 y / } \\
\text { females }\end{array}$} & \multirow{2}{*}{35} & \multirow{2}{*}{4.22} & No. & 2 & 14 & 37 & 24 & 51 & 5 \\
\hline & & & $\%$ & 1.5 & 10.53 & 27.82 & 18.05 & 38.34 & 3.76 \\
\hline \multirow{2}{*}{$\begin{array}{l}5-6 \text { y / } \\
\text { males }\end{array}$} & \multirow{2}{*}{48} & \multirow{2}{*}{5.27} & No. & 2 & 17 & 30 & 52 & 88 & 43 \\
\hline & & & $\%$ & 0.86 & 7.33 & 12.93 & 22.41 & 37.93 & 18.54 \\
\hline \multirow{2}{*}{$\begin{array}{c}5-6 \text { y / } \\
\text { females }\end{array}$} & \multirow{2}{*}{38} & \multirow{2}{*}{5} & No. & 2 & 8 & 16 & 40 & 70 & 38 \\
\hline & & & $\%$ & 1.15 & 4.6 & 9.2 & 22.99 & 40.23 & 21.83 \\
\hline \multirow{4}{*}{\multicolumn{2}{|c|}{$\begin{array}{c}\text { Spearman's correlation be- } \\
\text { tween sex and caries sever- } \\
\text { ity }\end{array}$}} & \multirow{2}{*}{\multicolumn{2}{|c|}{ correlation }} & - & - & - & - & - & - \\
\hline & & & & 0.693 & $0.891^{*}$ & 0.488 & 0.293 & 0.293 & 0.293 \\
\hline & & \multicolumn{2}{|c|}{$p$} & 0.127 & 0.017 & 0.326 & 0.573 & 0.573 & 0.573 \\
\hline & & \multicolumn{2}{|c|}{ No. } & 6 & 6 & 6 & 6 & 6 & 6 \\
\hline
\end{tabular}

* correlation is significant at the 0.05 level; dmft: decayed, missing and filled teeth; $\mathrm{d} 1, \mathrm{~d} 2, \mathrm{~d} 3, \mathrm{~d} 4 . \mathrm{d} 5$ and d6: caries severity 


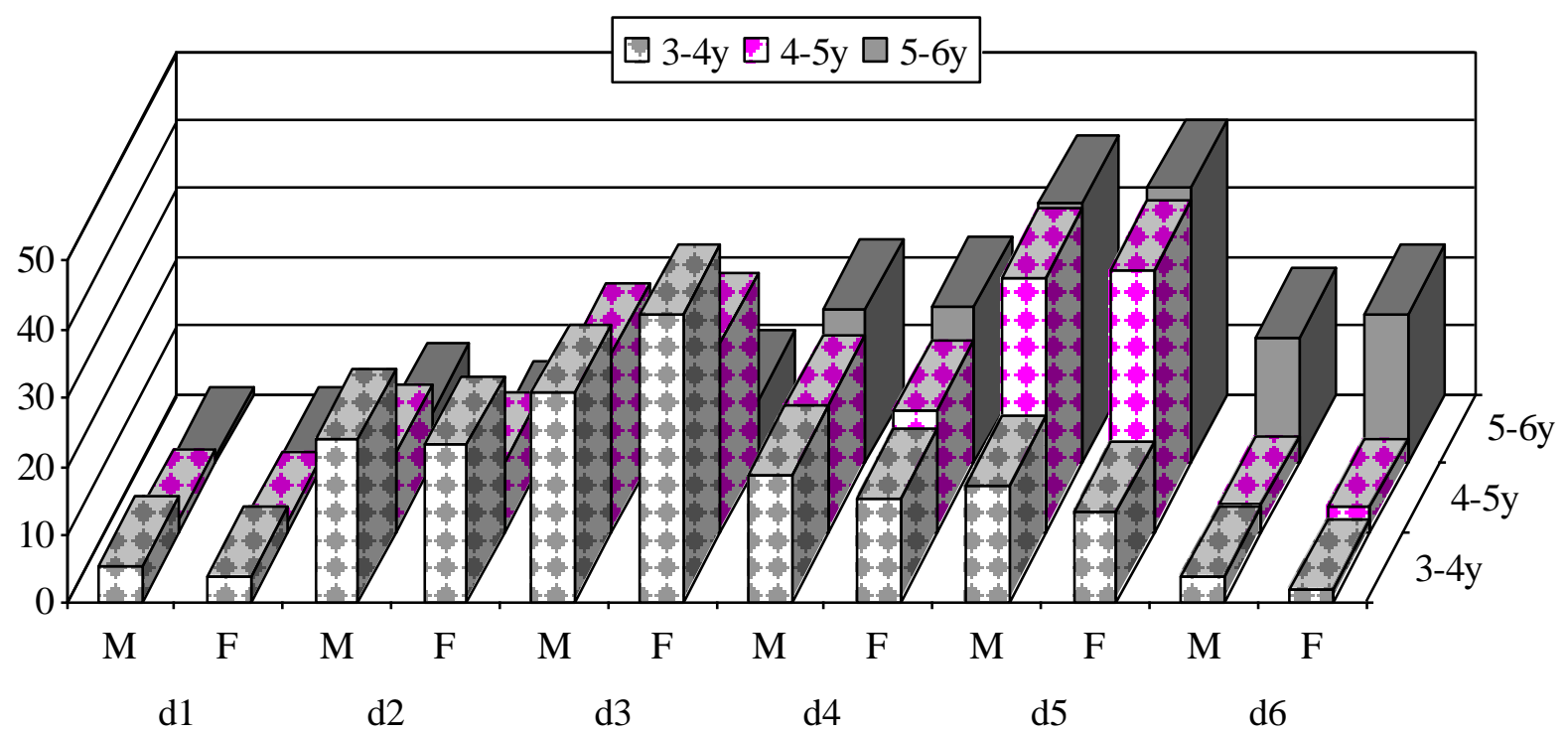

Figure (1): ICDAS gender differences.

M: males; F: females; d1, d2, d3, d4. d5 and d6: caries severity

Table (3) shows ICDAS among age groups. For group $(3-4 \mathrm{y})$, most of the children $(35.43 \%)$ were affected by (d3) caries severity followed by (d2) $(23.62 \%)$, and then (d4) (17.33\%). (d6) severity was presented in small percent $(3.15 \%)$. For age groups (4-5y) and (5-6y), (d5) severity was found in higher percent $(37.77 \%$ and $38.91 \%$ respectively) followed by (d3)
(26.98\%) for age group (4-5y) and (d4) $(22.66 \%)$ for age group (5-6y). (d1) severity was found in a very small percent $(1.8 \%)$ for age group (4-5y) and $(0.99 \%)$ for age group (5-6y). Spearman's correlation between age and caries severity was significant with (d1, d4, d5 and d6) at 0.001 level but not significant with (d2 and $\mathrm{d} 3$ ).

Table (3): ICDAS among age groups.

\begin{tabular}{|c|c|c|c|c|c|c|c|c|c|}
\hline Age & No. & dmft & No.\&\% & d 1 & d 2 & d 3 & d 4 & d 5 & d 6 \\
\hline \multirow[t]{2}{*}{ 3-4 y } & \multirow{2}{*}{59} & \multirow{2}{*}{2.28} & No. & 6 & 30 & 45 & 22 & 20 & 4 \\
\hline & & & $\%$ & 4.72 & 23.62 & 35.43 & 17.33 & 15.75 & 3.15 \\
\hline \multirow{2}{*}{ 4-5 y } & \multirow{2}{*}{74} & \multirow{2}{*}{4.17} & No. & 5 & 31 & 75 & 51 & 105 & 11 \\
\hline & & & $\%$ & 1.8 & 11.15 & 26.98 & 18.35 & 37.77 & 3.95 \\
\hline \multirow[t]{2}{*}{$5-6 y$} & \multirow{2}{*}{86} & \multirow{2}{*}{5.15} & No. & 4 & 25 & 46 & 92 & 158 & 81 \\
\hline & & & $\%$ & 0.99 & 6.16 & 11.33 & 22.66 & 38.91 & 19.95 \\
\hline \multirow{3}{*}{\multicolumn{3}{|c|}{$\begin{array}{c}\text { Spearman's correlation } \\
\text { between age and caries } \\
\text { severity }\end{array}$}} & correlation & $1.000^{* *}$ & 0.500 & 0.500 & $1.000 * *$ & $1.000 * *$ & $1.000 * *$ \\
\hline & & & $p$ & 0.000 & 0.667 & 0.667 & 0.000 & 0.000 & 0.000 \\
\hline & & & No. & 3 & 3 & 3 & 3 & 3 & 3 \\
\hline
\end{tabular}

** correlation is significant at the 0.01 level; dmft: decayed, missing and filled teeth; $\mathrm{d} 1, \mathrm{~d} 2, \mathrm{~d} 3, \mathrm{~d} 4 . \mathrm{d} 5$ and d6: caries severity

Regarding the relation between the level of parent's income and severity of dental caries (Table 4), for children with low income level $(<250000)$ of their parents, (d3) presented in larger percent (30.86\%) followed by (d5) $(28.4 \%)$ with small percent of (d1) $(2.47 \%)$. For the other three groups, (d5) severity was the most prominent $(29.65 \%, 37.94 \%$ and $36.7 \%$ respectively) and small percent of (d1) $(1.74 \%, 1.76 \%$ and $1.83 \%$ respectively). (d6) severity increased by increasing parent's income level $(4.93 \%, 9.3 \%, 13.24 \%$ and $14.22 \%$ ) respectively. Spearman's correlation between income level and caries severities was not significant. 
Table (4): Relation between income level and caries severity.

\begin{tabular}{|c|c|c|c|c|c|c|c|c|}
\hline Income level & No. & No.\&\% & d 1 & d 2 & d 3 & d 4 & d 5 & d 6 \\
\hline \multirow{2}{*}{$<250000 /$ month } & \multirow{2}{*}{24} & No. & 2 & 6 & 25 & 21 & 23 & 4 \\
\hline & & $\%$ & 2.47 & 7.41 & 30.86 & 25.93 & 28.4 & 4.93 \\
\hline \multirow{2}{*}{$<500000 /$ month } & \multirow{2}{*}{58} & No. & 3 & 20 & 40 & 42 & 51 & 15 \\
\hline & & $\%$ & 1.74 & 11.63 & 23.26 & 24.42 & 29.65 & 9.3 \\
\hline \multirow{2}{*}{$<1000000 /$ month } & \multirow{2}{*}{84} & No. & 6 & 32 & 63 & 65 & 129 & 45 \\
\hline & & $\%$ & 1.76 & 9.41 & 18.53 & 19.12 & 37.94 & 13.24 \\
\hline \multirow{2}{*}{$>1000000 /$ month } & \multirow{2}{*}{53} & No. & 4 & 28 & 38 & 37 & 80 & 31 \\
\hline & & $\%$ & 1.83 & 12.84 & 17.43 & 16.98 & 36.7 & 14.22 \\
\hline \multirow{3}{*}{$\begin{array}{c}\text { Spearman's correlation } \\
\text { between income level and } \\
\text { caries severity }\end{array}$} & \multicolumn{2}{|c|}{ correlation } & 0.800 & 0.800 & 0.400 & 0.400 & 0.800 & 0.800 \\
\hline & \multicolumn{2}{|r|}{$p$} & 0.200 & 0.200 & 0.600 & 0.600 & 0.200 & 0.200 \\
\hline & \multicolumn{2}{|r|}{ No. } & 4 & 4 & 4 & 4 & 4 & 4 \\
\hline
\end{tabular}

correlation is not significant; d1, d2, d3, d4. d5 and d6: caries severity

Regarding the relation between parent's education and dental caries (Table 5), for both (primary school) and (high education) groups, (d3) severity was the larger percent $(43.59 \%)$ and $(28.46 \%)$ respectively. While for the (secondary school) and (college) groups, (d5) severity pre- sented in larger percent $(35.42 \%)$ and $(38.22 \%)$ respectively. The larger percent of (d6) severity was related to (primary school) group (15.38\%), while less severity was related to (high education) group. Spearman's correlation was not significant.

Table (5): Relation between parent's education and caries severity.

\begin{tabular}{ccccccccc}
\hline Education & No. & No.\&\% & d 1 & d 2 & d 3 & d 4 & d 5 & d 6 \\
\hline Primary school & 7 & No. & 0 & 1 & 17 & 7 & 8 & 6 \\
& & $\%$ & 0.0 & 2.56 & 43.59 & 17.95 & 20.52 & 15.38 \\
Secondary school & 31 & No. & 2 & 12 & 32 & 33 & 51 & 14 \\
College & & $\%$ & 1.39 & 8.33 & 22.22 & 22.92 & 35.42 & 9.72 \\
& 137 & No. & 10 & 48 & 82 & 104 & 193 & 68 \\
High education & 44 & 1.98 & 9.5 & 16.24 & 20.59 & 38.22 & 13.74 \\
& & No. & 3 & 25 & 35 & 21 & 31 & 8 \\
Spearman's correlation & correlation & 0.800 & 0.800 & 0.800 & 0.400 & 0.400 & 0.400 \\
between parent's education & & $p$ & 0.200 & 0.200 & 0.200 & 0.600 & 0.600 & 0.600 \\
and caries severity & & No. & 4 & 4 & 4 & 4 & 4 & 4 \\
\hline
\end{tabular}

correlation is not significant; $d 1, d 2, d 3, d 4 . d 5$ and d6: caries severity

Table (6) shows the relation between dental attendance and caries severity. For the children who have previous dental attendance, (d3) severity presented in a higher percent $(30.12 \%)$ followed by (d4) (27.8\%), (d5) (20.85\%), (d2) (14.29\%), (d6) (4.62\%) and finally (d1) (2.32\%). For the other group, (d5) severity was obvious $(41.49 \%)$ followed by $(\mathrm{d} 4)(16.85 \%),(\mathrm{d} 3)$ $(15.94 \%),(\mathrm{d} 6)(15.22 \%),(\mathrm{d} 2)(8.87 \%)$ and finally (d1) (1.63\%). Spearman's correlation was significant at 0.001 level. 
Table (6): Relation between dental attendance and caries severity.

\begin{tabular}{ccccccccc}
\hline Attendance & No. & No.\&\% & d 1 & d 2 & d 3 & d 4 & d 5 & d 6 \\
\hline Visited a dentist & 84 & No. & 6 & 37 & 78 & 72 & 54 & 12 \\
Did not visit & $\%$ & 3.32 & 14.29 & 30.12 & 27.8 & 20.85 & 4.62 \\
& & No. & 9 & 49 & 88 & 93 & 229 & 84 \\
\hline $\begin{array}{c}\text { Spearman's correla- } \\
\text { tion between dental }\end{array}$ & correlation & $1.000^{* *}$ & $1.000^{* *}$ & $1.000^{* *}$ & $1.000^{* *}$ & $1.000 * *$ & $1.000 * *$ \\
attendance and caries & $p$ & 0.000 & 0.000 & 0.000 & 0.000 & 0.000 & 0.000 \\
severity & No. & 2 & 2 & 2 & 2 & 2 & 2 \\
\hline$* *$ correlation is significant at the 0.01 level; d1, d2, d3, d4. d5 and d6: caries severity & &
\end{tabular}

About the relation between the cause of dental attendance and caries severity (table 7), for the group (Pain / Treatment), (d4) severity was the larger percent $(32.14 \%)$ with a small percent of (d1) $(1.79 \%)$. For the (Prevention) group, there was no (d6) severity; the larger percent was (d2) (47.83\%). For (Restoration) group, (d3) severity was the larger percent $(37.31 \%)$. For (Extraction) group, there was no (d1) severity; the larger percent was (d4) (35.09\%). Spearman's correlation was not significant.

Table (7): Relation between causes of dental attendance and caries severity.

\begin{tabular}{|c|c|c|c|c|c|c|c|c|}
\hline Causes of attendance & No. & $\begin{array}{c}\text { No.\& } \\
\%\end{array}$ & d 1 & d 2 & d 3 & d 4 & d 5 & d 6 \\
\hline \multirow{2}{*}{ Pain / treatment } & \multirow{2}{*}{33} & No. & 2 & 10 & 34 & 36 & 27 & 3 \\
\hline & & $\%$ & 1.79 & 8.93 & 30.36 & 32.14 & 24.11 & 2.67 \\
\hline \multirow{2}{*}{ Prevention } & \multirow{2}{*}{12} & No. & 1 & 11 & 7 & 3 & 1 & 0 \\
\hline & & $\%$ & 4.35 & 47.83 & 30.43 & 13.04 & 4.35 & 0.0 \\
\hline \multirow{2}{*}{ Restoration } & \multirow{2}{*}{23} & No. & 3 & 9 & 25 & 13 & 12 & 5 \\
\hline & & $\%$ & 4.48 & 13.43 & 37.31 & 19.4 & 17.91 & 7.47 \\
\hline \multirow{2}{*}{ Extraction } & \multirow{2}{*}{16} & No. & 0 & 7 & 12 & 20 & 14 & 4 \\
\hline & & $\%$ & 0.0 & 12.28 & 21.05 & 35.09 & 24.56 & 7.02 \\
\hline \multirow{3}{*}{$\begin{array}{c}\text { Spearman's correlation } \\
\text { between attendance } \\
\text { causes and caries se- } \\
\text { verity }\end{array}$} & \multicolumn{2}{|c|}{ correlation } & $\begin{array}{c}- \\
0.400\end{array}$ & $\begin{array}{c}- \\
0.800\end{array}$ & $\begin{array}{c}- \\
0.400\end{array}$ & $\begin{array}{c}- \\
0.200\end{array}$ & $\begin{array}{c}- \\
0.200\end{array}$ & 0.600 \\
\hline & \multicolumn{2}{|r|}{$p$} & 0.600 & 0.200 & 0.600 & 0.800 & 0.800 & 0.400 \\
\hline & \multicolumn{2}{|c|}{ No. } & 4 & 4 & 4 & 4 & 4 & 4 \\
\hline
\end{tabular}

Table (8) shows the relation between tooth brushing practice and dental caries severity. For the children who did not brush their teeth, (d5) severity was presented in larger percent $(41.95 \%)$ followed by (d4) $(20.25 \%)$, (d3) $(15.73 \%)$, (d6) $(14.11 \%),(\mathrm{d} 2)(6.87 \%)$ and finally (d1) $(1.09 \%)$. For the other group, (d3) severity was presented in a larger percent $(30.62 \%)$ followed by (d4) $(20.54 \%),(d 5)$ (19.77\%), (d2) (18.6\%), (d6) (6.98\%) and finally (d1) (3.49\%). Spearman's correla- tion was significant at (0.001) level with (d1) and (d2) but not significant with the other severities. About the frequency of teeth brushing, for all the three groups, (d3) severity was presented in a large percent and was increased from (infrequent brushing) (27.89\%) to (once daily) $(37.74 \%)$ and to (twice daily) (40\%). While (d6) severity was reduced from (infrequently) (8.43\%) to (once daily) $(3.77 \%)$ and disappeared for (twice daily) 
group. Spearman's correlation was signifi-

cant at (0.001) level.

Table (8): Relation between tooth brushing practice and caries severity.

\begin{tabular}{|c|c|c|c|c|c|c|c|c|}
\hline Brushing practice & No. & No.\&\% & d 1 & d 2 & d 3 & d 4 & d 5 & d 6 \\
\hline \multirow{2}{*}{ Do not brush } & \multirow{2}{*}{122} & No. & 6 & 38 & 87 & 112 & 232 & 78 \\
\hline & & $\%$ & 1.09 & 6.87 & 15.73 & 20.25 & 41.95 & 14.11 \\
\hline \multirow{2}{*}{ Brush } & \multirow{2}{*}{97} & No. & 9 & 48 & 79 & 53 & 51 & 18 \\
\hline & & $\%$ & 3.49 & 18.6 & 30.62 & 20.54 & 19.77 & 6.98 \\
\hline \multirow{2}{*}{ Infrequently } & \multirow{2}{*}{68} & No. & 5 & 32 & 53 & 41 & 43 & 16 \\
\hline & & $\%$ & 2.63 & 16.84 & 27.89 & 21.58 & 22.63 & 8.43 \\
\hline \multirow{2}{*}{ Once daily } & \multirow{2}{*}{22} & No. & 3 & 12 & 20 & 9 & 7 & 2 \\
\hline & & $\%$ & 5.66 & 22.64 & 37.74 & 16.98 & 13.21 & 3.77 \\
\hline \multirow{2}{*}{$\begin{array}{l}\text { Twice daily } \\
\text { and more }\end{array}$} & \multirow{2}{*}{7} & No. & 1 & 4 & 6 & 3 & 1 & 0 \\
\hline & & $\%$ & 6.67 & 26.66 & 40 & 20 & 6.67 & 0.0 \\
\hline \multirow{3}{*}{$\begin{array}{l}\text { Spearman's correla- } \\
\text { tion between brush- } \\
\text { ing practice and ca- } \\
\text { ries severity }\end{array}$} & \multicolumn{2}{|c|}{ correlation } & $1.000^{* *}$ & $1.000 * *$ & -1.000 & -1.000 & -1.000 & -1.000 \\
\hline & \multicolumn{2}{|r|}{$p$} & 0.00 & 0.00 & 1.000 & 1.000 & 1.000 & 1.000 \\
\hline & \multicolumn{2}{|r|}{ No. } & 2 & 2 & 2 & 2 & 2 & 2 \\
\hline \multirow{3}{*}{$\begin{array}{l}\text { Spearman's correla- } \\
\text { tion between brush- } \\
\text { ing frequency and } \\
\text { caries severity }\end{array}$} & \multicolumn{2}{|c|}{ correlation } & $\begin{array}{c}- \\
1.000^{* *}\end{array}$ & $\begin{array}{c}- \\
1.000 * *\end{array}$ & $\frac{-}{1.000^{* *}}$ & $\overline{-}^{-}$ & $\overline{-}^{-}$ & $\overline{-}^{-}$ \\
\hline & \multicolumn{2}{|r|}{$p$} & 0.00 & 0.00 & 0.00 & 0.00 & 0.00 & 0.00 \\
\hline & \multicolumn{2}{|r|}{ No. } & 3 & 3 & 3 & 3 & 3 & 3 \\
\hline
\end{tabular}

** correlation is significant at the 0.01 level; $\mathrm{d} 1, \mathrm{~d} 2, \mathrm{~d} 3, \mathrm{~d} 4$. $\mathrm{d} 5$ and d6: caries severity

The distribution of decayed, missing and filled teeth and caries free children according to age and gender were showed in table (9) and figure (2). The dmft value was clearly increased by increasing the age for both males and females $\left(3-4 y, \delta^{\lambda}\right.$ : $2.42 \%, 3-4 y$, $9: 2.11 \%, 4-5 y, o^{\top}: 4.12 \%$, 4-5y, ㅇ: $4.22 \%, 5-6 y, 0$ : $5.27 \%, 5-6 y$, ․ $5 \%$ ). Decayed teeth were formed the major component of $\mathrm{dmft}$ index for all the three age groups. The percentage of decayed teeth were larger for the age group (3-4y) $(93.75 \%$ for males and $94.55 \%$ for females) followed by age group (5-6y) (91.7\% for males and $91.58 \%$ for females), and then the age group (4-5y) (90.06\% for males and $89.86 \%$ for females). A small percentage of the decayed teeth were treated by restoration of any type (between 5\%-8.11\%). The percentage of filled teeth for the age group (4-5y) was larger than the other two groups for both genders. The percentages of filled teeth were slightly larger for females than the males. While extracted teeth were increased by increasing the age and the percentage for males were slightly larger than for females except for age group (5-6y), the percentages were equal. The percentages of caries free teeth were reduced by increasing the age from $(30.3 \%)$ for age group (3-4y) males to (18.42\%) for age group (5-6y) females. Spearman's correlation between age and (dmft, decayed and missing) was significant at 0.01 level, but not significant with (filling and caries free). Spearman's correlation with the gender was not significant. 
Table (9): Distribution of decayed, missing and filled teeth.

\begin{tabular}{|c|c|c|c|c|c|c|c|c|}
\hline Age & Sex & No. & No.\&\% & $\mathrm{dmft}$ & decayed & missing & filled & $\begin{array}{c}\text { Caries } \\
\text { free }\end{array}$ \\
\hline \multirow{6}{*}{$3-4 y$} & \multirow{2}{*}{ male } & \multirow{2}{*}{33} & No. & 80 & 75 & 1 & 4 & 10 \\
\hline & & & $\%$ & 2.42 & 93.75 & 1.25 & 5 & 30.3 \\
\hline & \multirow{2}{*}{ female } & \multirow{2}{*}{26} & No. & 55 & 52 & 0 & 3 & 8 \\
\hline & & & $\%$ & 2.11 & 94.55 & 0.0 & 5.45 & 30.77 \\
\hline & \multirow{2}{*}{ total } & \multirow{2}{*}{59} & No. & 135 & 127 & 1 & 7 & 18 \\
\hline & & & $\%$ & 2.29 & 94.07 & 0.74 & 5.19 & 30.5 \\
\hline \multirow{6}{*}{$4-5 y$} & \multirow{2}{*}{ male } & \multirow{2}{*}{39} & No. & 161 & 145 & 4 & 12 & 11 \\
\hline & & & $\%$ & 4.12 & 90.06 & 2.48 & 7.46 & 28.21 \\
\hline & \multirow{2}{*}{ female } & \multirow{2}{*}{35} & No. & 148 & 133 & 3 & 12 & 10 \\
\hline & & & $\%$ & 4.22 & 89.86 & 2.03 & 8.11 & 28.57 \\
\hline & \multirow{2}{*}{ total } & \multirow{2}{*}{74} & No. & 309 & 278 & 7 & 24 & 21 \\
\hline & & & $\%$ & 4.17 & 89.97 & 2.26 & 7.77 & 28.38 \\
\hline \multirow{6}{*}{$5-6 y$} & \multirow{2}{*}{ male } & \multirow{2}{*}{48} & No. & 253 & 232 & 8 & 13 & 12 \\
\hline & & & $\%$ & 5.27 & 91.7 & 3.16 & 5.14 & 25 \\
\hline & \multirow{2}{*}{ female } & \multirow{2}{*}{38} & No. & 190 & 174 & 6 & 10 & 7 \\
\hline & & & $\%$ & 5 & 91.58 & 3.16 & 5.26 & 18.42 \\
\hline & \multirow{2}{*}{ total } & \multirow{2}{*}{86} & No. & 443 & 406 & 14 & 23 & 19 \\
\hline & & & $\%$ & 5.15 & 91.65 & 3.16 & 5.19 & 22.09 \\
\hline \multirow{3}{*}{\multicolumn{2}{|c|}{$\begin{array}{c}\text { Spearman's correlation } \\
\text { with age }\end{array}$}} & \multicolumn{2}{|c|}{ correlation } & $0.956^{* *}$ & $0.956 * *$ & $0.956 * *$ & 0.728 & 0.182 \\
\hline & & \multicolumn{2}{|r|}{$\mathrm{P}$} & 0.003 & 0.003 & 0.003 & 0.101 & 0.730 \\
\hline & & \multicolumn{2}{|c|}{ No. } & 6 & 6 & 6 & 6 & 6 \\
\hline \multirow{3}{*}{\multicolumn{2}{|c|}{$\begin{array}{l}\text { Spearman's correlation } \\
\text { with gender }\end{array}$}} & \multicolumn{2}{|c|}{ Correlation } & -0.293 & -0.293 & -0.293 & $\begin{array}{c}- \\
0.396\end{array}$ & -0.792 \\
\hline & & \multicolumn{2}{|r|}{$\mathrm{P}$} & 0.573 & 0.573 & 0.573 & 0.437 & 0.060 \\
\hline & & \multicolumn{2}{|c|}{ No. } & 6 & 6 & 6 & 6 & 6 \\
\hline
\end{tabular}




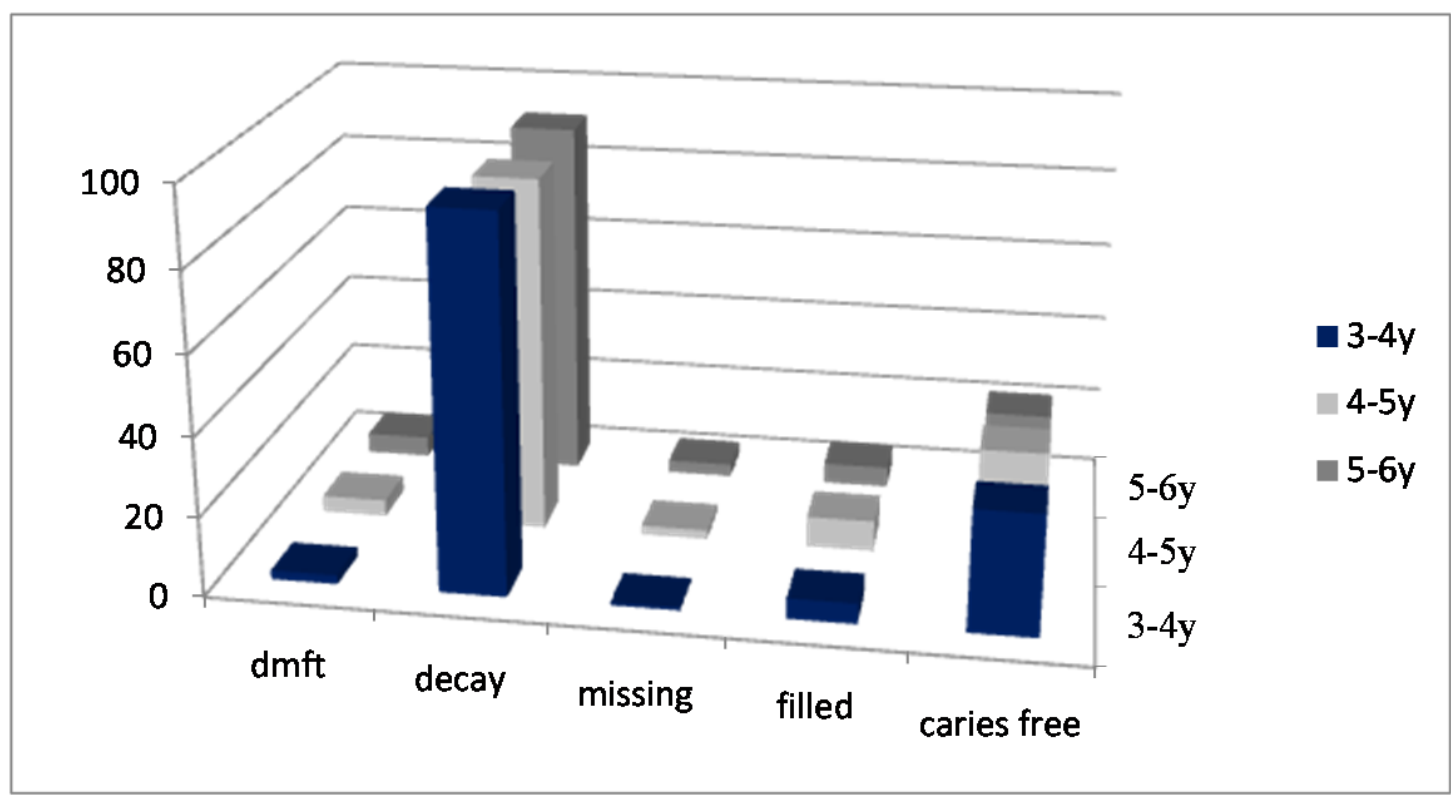

Figure (2): Distribution of decayed, missing and filled teeth and caries free children. dmft: decayed, missing and filled teeth

\section{DISCUSSION}

This study demonstrated early initiation of tooth decay in preschool children confirming previous observations ${ }^{(9-11)}$ and this increase by increasing the age from 3 to 6 years due to accumulative nature of carious process, which is agreed with other studies. ${ }^{(12,13)}$ The other usual observation obtained, is that the mean dmft for boys is greater than for girls which is also agreed with many studies. ${ }^{(13-16)}$ This is due to the fact that females care with their health and appearance more than males even at small ages. Regarding the component of $\mathrm{dmft}$ index, it is normally to find a marked influence of $\mathrm{d}$ component compared with $\mathrm{m}$ and $\mathrm{f}$, because decay starts at this age and remains untreated until the child reaches a larger age. Younger children present more behavioral problems in dental clinics and this could be the reason why the number of filled teeth in these children was lower compared to the older group. In the same way, caries severity increase by increasing age and without sufficient necessary filling result in tooth extraction at older age. This is agreed with other studies. ${ }^{(9,15,17)}$

Studies in many parts of the worlds have consistently recorded that parents' income level and education are strongly associated with dental caries prevalence in children. ${ }^{(7,11,12,18)}$ Studies ${ }^{(11,16,18)}$ found that increasing parents' education and in- come level are associated with decreasing caries severity. In this study, children of parents with high income level had more (d6) caries severity than the other groups, this may due to a large amount of refined sugary food and confectionary or that their parents were busy with work and have no enough time to take their children to dental clinic to have their teeth restored. The income of many people has increased greatly within a short time interval and their life styles and value systems may well have altered radically in consequence.

Regarding dental attendance, it is not unusual to find that children who did not attend a dental treatment were presented with more caries severity. Here, we can clearly detect the effect of early attendance to check for initial stages of carious process, and the superior influence of preventive measures over all the other treatments. As we observed, the children who had previous attendance for preventive measures had zero (d6) severity and small percent of (d5) severity.

A cost-effective and scientifically supported way of caries prevention is to encourage parents to brush the teeth of their children using fluoride tooth paste twice daily, starting at an early age. Tooth brushing by parents can remove dental plaque more effectively and such children are more likely to be caries free than those 
who brush by themselves.

The value of mean dmft that is obtained when compared with that from other Iraqi studies, the results were different, For example: (1.96) ${ }^{(19)}$ and $(3.79)^{(20)}$ at age 6 years and $(2.63)^{(21)}$ and $(6.82)^{(22)}$ at age 4-6 years. For other countries: (5.7) at age 6 years in Ukraine, ${ }^{(23)}(2.9)$ at age 3-4 years in Brazil, ${ }^{(18)}(3.5)$ at 3 years and (4.8) at 5 years in Canada, ${ }^{(13)}(1.4)$ at age 4-6 years in North Brisbane-Australia, ${ }^{(16)}$ (4.03) at age 6 years in Al-Ahsaa, Saudi Arabia $^{(14)}$ and (7.34) at 6-7 years in Riyadh, Saudi Arabia. ${ }^{(24)}$

The percentages of caries free children were low and continue to decrease by increasing the age, which in turn associated with increasing $\mathrm{dmft}$ value. The percentages of caries free children in other Iraqi studies were different. Example: (25.8\%) ${ }^{(19)}$ and $(16.66 \%)^{(20)}$ at age 6 years, and $(13.75 \%)^{(21)}$ and $(16.87 \%)^{(22)}$ at age 4-6 years. For other countries: $(5.4 \%)$ in Bra$\mathrm{zil}^{(18)}(17.2 \%)$ in Thailand, ${ }^{(7)}(5 \%-6 \%)$ in Riyadh, ${ }^{(24)}$ and $(66.3 \%)$ in North Brisbane. ${ }^{(16)}$

\section{CONCLUSIONS}

The study has provided useful baseline data for future comparisons. Furthermore, the caries prevalence and severity information would assist in the determination of treatment needs and preventive efforts required in this population. The knowledge of caries pattern would help clinicians in the formulation of appropriate treatment strategies. It could be deduced from the above results and discussion that considerable efforts are required in prevention of dental caries in these children. There is a lot of untreated caries, which needs immediate attention. It is proposed that a school dental service may be initiated in the area. The service could serve dual purpose in the area i.e. provision of preventive dental services and restorative care to children.

\section{REFERENCES}

1. Saleh KM. Dental caries and treatment needs of primary and permanent teeth for children attending pedodontic clinic. AlRafidain Dent J. 2007; 7(1): 80-87.

2. Khamrco TY. Acomparative study of oral health status among urban and rural school students in Ninevah Governorate-Iraq. $A l$ Rafidain Dent J. 2001; 1: 7-15.

3. Al-Naimi RJ. Dental caries experience of rural and urban school children in Ninevah Province. A comparative study. AlRafidain Dent J. 2001; 1: 18-23.

4. Rodrigues CS and Sheiham A. the relationship between dietary guide lines, sugar intake and caries in primary teeth in low income Brazilian 3 years old: a longitudinal study. Int J Paediatr Dent. 2000; 10: 47-55.

5. Wennhall I, Matsson L, Schroder U, Twetman S. Caries prevalence in 3-years old children in a low socioeconomic multicultural urban area in Southern Sweden. Swed Dent J. 2002; 26: 166-172.

6. Vanobbergen J, Matsen L, Lesaffre E, Bogaerts K, Declerck D. Assessing risk indicators for dental caries in the primary dentition. Comm Dent Oral Epidemiol. 2001; 29: 424-434.

7. Vachirarojpisan T, Shinada K, Kawaguchi Y, Laungwechagan P, Somkote T, Detsomboonrat P. Early childhood caries in children aged 6-19 months. Comm Dent Oral Epidemiol. 2004; 32:133-142.

8. International caries detection and assessment system coordinating committee. Rationale and evidence for the International Caries Detection and Assessment System (ICDAS II). In:stookey G, editor, proceedings of the 2005 Annual Indiana Conference, July 6-9,2005. Indianapolis, IN: Indiana University School of Dentistry; 2006 in press.

9. Salman FD, Gasgoos SS and Qasim AA. The effectiveness of dental health education program on oral health status in nursery school children. The fifth scientific conference, College of Nursing, University of Mosul, 2008: 80-89.

10.Petersen PE. Effect of school-based oral health education programme in Wuhan city, people republic of China. Inter Dent J. 2004; 54: 33-41.

11.Pereira MBB and Freire MCM. An infant oral health program in Goiania-Go, Brazil: results after 3 years of establishment. Braz Oral Res. 2004; 18(1): 12-17.

12.Al-Malik MI, Holt RD, Bedi R. Erosion, caries and rampant caries in preschool children in Jeddah, Saudi Arabia. Community Dent Oral Epidemiol. 2002; 30: 1623. 
13.Peressini S, Leake L, Mayhall JT, Maar M and Trudeau R. Prevalence of early childhood caries among First Nations children, District of Manitoulin, Ontario. . Int $J$ Paediatr Dent. 2004; 14: 101-110.

14.Al-Shalan TA. Saudi parents Knowledge of and attitude toward the prevention of dental caries. Saudi Dent J. 2003; 15(1): 27-32.

15.Al-Wazzan KA. Dental caries prevalence in 6-7 year-old school children in Riyadh region: A comparative study with the 1987Oral Health Survey of Saudi Arabia Phase I. Saudi Dent J. 2004; 16(2): 54-60.

16. Hallette KB and O'Rourke PK. Dental caries experience of preschool children from the north Brisbane region. Aust Dent J. 2002; 47(4): 331-338.

17.Wyne AH, Al-Ghorabi BM, Al-Asiri YA, Khan NB. Caries prevalence in Saudi primary school children of Riyadh and their teachers' oral health knowledge, attitude and practices. Saudi Med J. 2002; 23: 77 81.

18.Dini EL, Holt RD and Bedi R. Caries and its association with infants feeding and oral health-related behaviours in 3-4-yearold Brazilian children. Community Dent Oral Epidemiol. 2000; 28: 241-248.
19. Khamrco TY, Makani LA, Jazrawi KH. Dental caries changes between (1989) and (2001) in children aged 3-14 years in Kasa Fakhra and Al-Shamsiat villages, Ninevah Governorate. Iraq. Al-Rafidain Dent $J$. 2002; 2(sp-Iss): 269-278.

20.Kamrco TY and Salman FD. Prevalence of dental caries among primary school children age 6-12 years old in Mosul City center/ Ninevah. Iraqi Dent J. 2001; 27: 6582.

21.Kamrco TY and Al-Naimi RJ. Caries experience and treatment needs of primary dentition in Sharkhan villages. Al-Rafidain Dent J. 2001; 2: 174-179.

22.Jazrawi KH. Evaluation of dental caries prevalence among kindergarten's children in Mosul City center. Al-Rafidain Dent J. 2009; 9(1): 120-130.

23.Spivak K, Hayes $\mathrm{C}$ and Maguire JH. Caries prevalence, oral health behavior, and attitudes in children residing in radiation-contaminated and- non contaminated towns in Ukraine. Community Dent Oral Epidemiol. 2004; 32: 1-9.

24.Al-Dosari AM, Wyne AH, Akpata ES and Khan NB. Caries prevalence among secondary school children in Riyadh and Qaseem. Saudi Dent J. 2003: 15(2): 96-99. 